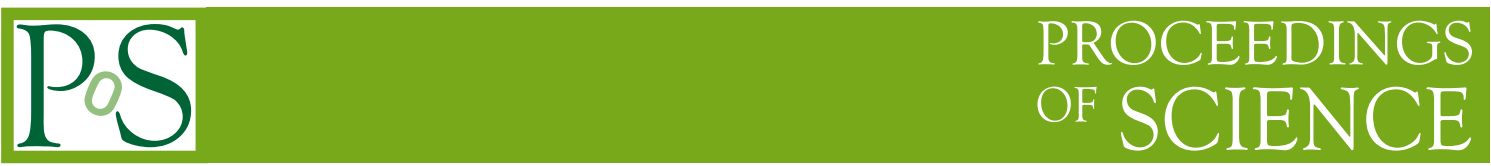

\title{
Search for Dark Matter with GLAST
}

\author{
Aldo Morselli* \\ INFN Roma 2 \\ Roma. Italy \\ E-mail: aldo.morselli@roma2.infn.it
}

The direct detection of annihilation products in cosmic rays offers an alternative way to search for supersymmetric dark matter particles candidates. The study of the spectrum of gamma-rays, antiprotons and positrons in space has already showed some deviation from the expected signals but with weak statistical evidence. We will review the present situation and the achievable limits with the GLAST experiment.

GLAST [1] is a next generation high-energy gamma-ray observatory designed for making observations of celestial gamma-ray sources in the energy band extending from $20 \mathrm{MeV}$ to more than $300 \mathrm{GeV}$. The principal instrument of the GLAST mission is the Large Area Telescope (LAT) that is being developed as a mission involving an international collaboration of particle physics and astrophysics communities from 26 institutions in the United States, Italy, Japan, France and Germany. The main scientific objects are the study of all gamma ray sources such as blazars, gamma-ray bursts, supernova remnants, pulsars, diffuse radiation, and unidentified high-energy sources. The main characteristics of the detector, extensively studied with Monte Carlo and beam tests, are an energy range between $20 \mathrm{MeV}$ and $300 \mathrm{GeV}$, a field of view of $\sim 3 \mathrm{sr}$, an energy resolution of $\sim 5 \%$ at $1 \mathrm{GeV}$, a point source sensitivity of $2 \times 10^{-9}\left(\mathrm{ph} \mathrm{cm}^{-2} \mathrm{~s}^{-1}\right)$ at $0.1 \mathrm{GeV}$, an event deadtime of $20 \mathrm{~ms}$ and a peak effective area of $10000 \mathrm{~cm}^{2}$, for a required power of $600 \mathrm{~W}$ and a payload weight of $3000 \mathrm{Kg}$ [2].

International Europhysics Conference on High Energy Physics

July 21st - 27th 2005

Lisboa, Portugal

${ }^{*}$ Speaker. 


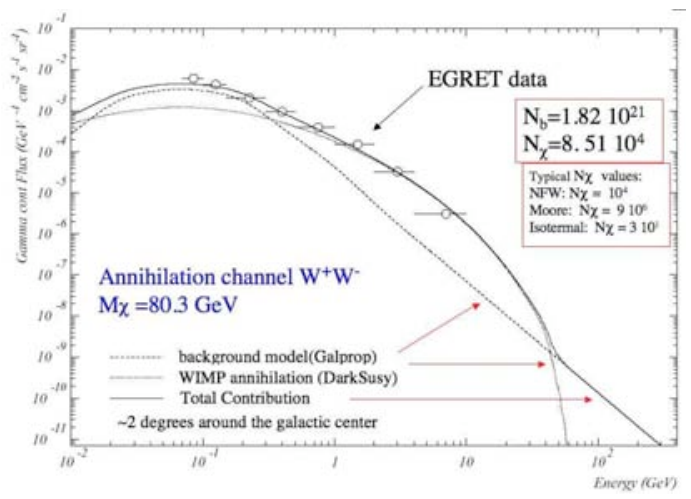

Figure 1: Fit of the EGRET Galactic Center $\mathbf{g}$ ray data for a sample WIMP models with $M_{C}=$ $80.3 \mathrm{GeV}$ and $W^{-} W^{+}$annihilation channel.

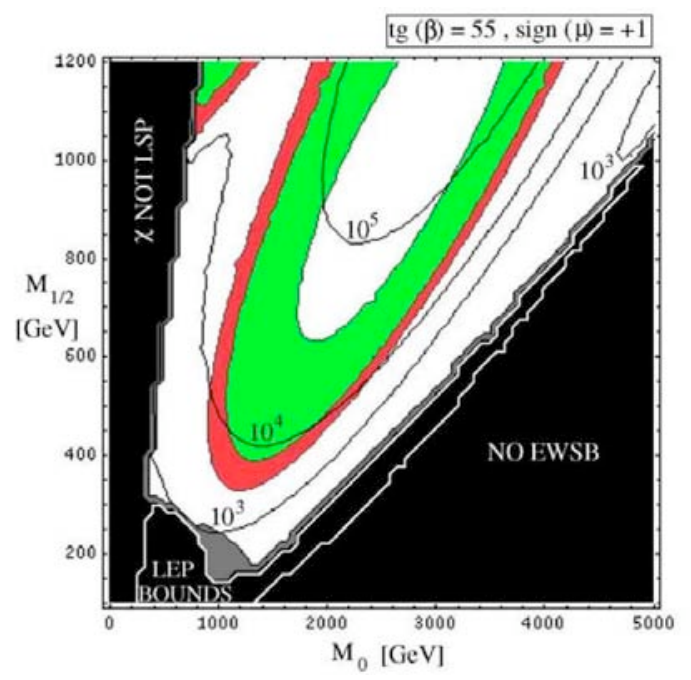

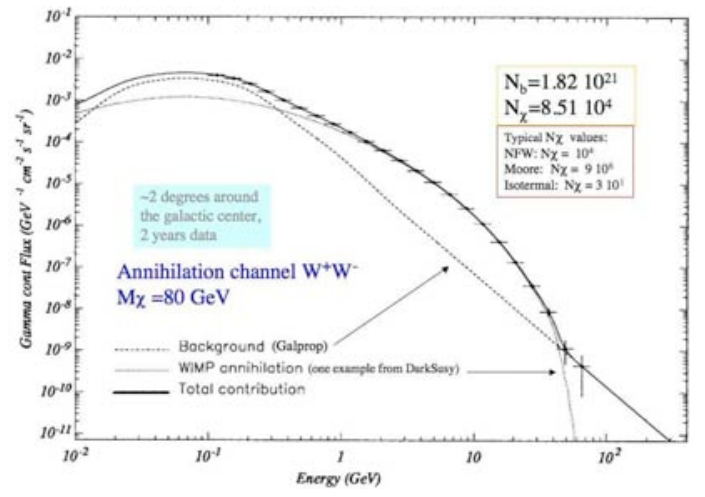

Figure 2: The same fluxes with the kind of statistical errors that it is expected in three years with GLAST

Figure 3: Contour plot in the mSUGRA $\left(m_{0}, m_{1 / 2}\right)$ plane, for the value of the normalization factor $N_{C}$, that allows the detection of the neutralino $g$ ray signal with GLAST. In the green region $0.13 \leq$ $\Omega_{c} h^{2} \leq 1$, while the red region corresponds to the WMAP range $0.09 \leq \Omega_{c} h^{2} \leq 0.13$. The black region corresponds to models that are excluded either by incorrect EWSB, LEP bounds violations or because the neutralino is not the LSP. In the dark shaded region $m_{h_{0}}<114.3 \mathrm{GeV}$ and $h_{0}$ is the lightest Higgs.

\section{The GLAST experiment}

GLAST could be of particular interest for the search of dark matter candidates. Figure 1 shows the EGRET data located within $2^{\circ}$ the Galactic center together with the diffuse gamma ray background flux expected from the standard interactions and propagation models of cosmic ray protons and electrons and an example of the flux due to neutralino annihilation in the dark matter halo [3].

Figure 2 shows the same fluxes of figure 1 with the kind of statistical errors that is expected in two years with GLAST [4]. It can be seen that GLAST will have the necessary statistical and energetic accuracy to distinguish the two kind of spectral shape.

So we have performed a complete study in the minimal supergravity (mSUGRA) framework. Figure 3 show the GLAST capability for $\tan b=55$ to probe in two years the supersymmetric dark matter hypothesis. Other examples with different values of $\tan b$ can be found in [4] and a complementary analysis with the PAMELA experiment [5] can be found in [6]. 


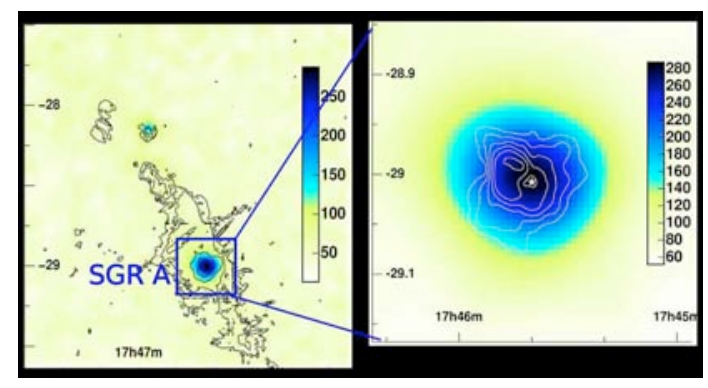

Figure 4: The galactic center as seen by the H.E.S.S. experiment (F. Aharonian et al., 2004). The source position is consistent with SGR A* to $6 "$ and slightly extended

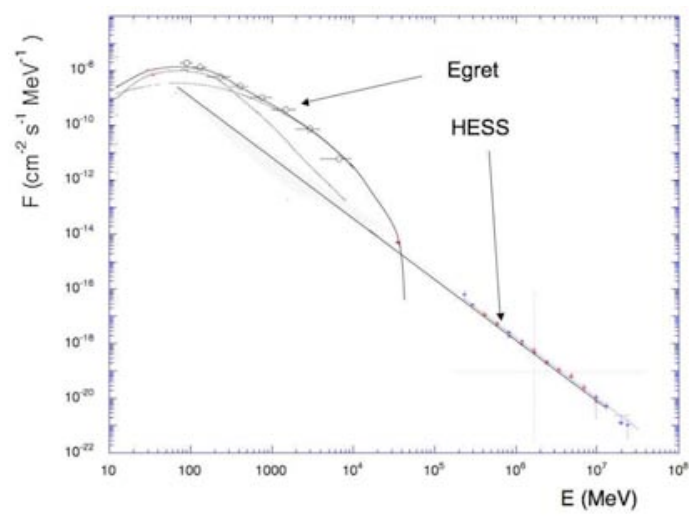

Figure 5: Extrapolation of the H.E.S.S flux to the EGRET energies if one use the same spectral index measured by H.E.S.S.

Recentry the H.E.S.S. experiment has discovered a powerful $\mathrm{TeV}$ gamma-ray source in the galactic center (see figure 4 and ref. [7]. The source position is consistent with SGR A* to 6" and slightly extended (see figure 4) with an unbroken power-law with $\Gamma=2.2$ and no evidence for variability on a variety of time scales.

There have been studies to connect the H.E.S.S. flux with neutralino annihilation [8] but as can be noted from figure 5, the extrapolation of the H.E.S.S flux to the EGRET energies with the same power law give a flux that is a factor hundred less and then it is very likely that the two sources are different.

\section{References}

[1] E. Bloom, G. Godfrey, S. Ritz, (1998), Proposal for the Gamma-ray Large Area Telescope, SLAC-R22.

A. Morselli (1997), XXXIInd Rencontres de Moriond, Very High Energy Phenomena in the Universe, Les Arc, France, January 18-25, 1997, Editions Frontiers, p.123

[2] A. Morselli, (2002) "Astroparticle and Gamma ray Physics in Space", Frascati Physics Series Vol.XXIV , pp. 363-380, http://www.roma2.infn.it/infn/aldo/ISSS01.html

R.Bellazzini et al. (2002), Nuclear Physics B , 113B, 303-309

A.Morselli (2004) Nuclear Inst.and Methods in Physics Research A 530, 158-162

[3] A.Morselli, A.Lionetto, A.Cesarini, F.Fucito, P.Ullio, (2002) Nuclear Physics 113B 213-220

[4] A.Cesarini, F.Fucito, A.Lionetto, A.Morselli and P.Ullio, (2004) Astropart. Phys. 21 (2004) 267 [astro-ph/0305075].

[5] P.Picozza, A.Morselli, (2003) J. Phys. G: Nucl. Part. Phys., 29, 903-911

[6] A.Lionetto, A.Morselli, V.Zdravkovic (2005), J. Cosmol. Astropart. Phys. JCAP09, 010 [astro-ph/0502406]

[7] F. Aharonian et al. (2004) [The HESS Collaboration], arXiv:astro-ph/040814

[8] S.Profumo, astro-ph/0508628

L.Bergstrom, T.Bringmann, M.Eriksson, M. Gustafsson (2005) hep-ph/0507229 